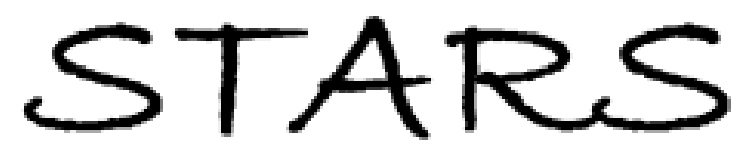

University of Central Florida

STARS

$1-1-2003$

\title{
Complex structure of carbon nanotubes and their implications for formation mechanism
}

\author{
Dan Zhou \\ University of Central Florida \\ Lee Chow \\ University of Central Florida
}

Find similar works at: https://stars.library.ucf.edu/facultybib2000 University of Central Florida Libraries http://library.ucf.edu

This Article is brought to you for free and open access by the Faculty Bibliography at STARS. It has been accepted for inclusion in Faculty Bibliography 2000 s by an authorized administrator of STARS. For more information, please contact STARS@ucf.edu.

\section{Recommended Citation}

Zhou, Dan and Chow, Lee, "Complex structure of carbon nanotubes and their implications for formation mechanism" (2003). Faculty Bibliography 2000s. 4156.

https://stars.library.ucf.edu/facultybib2000/4156

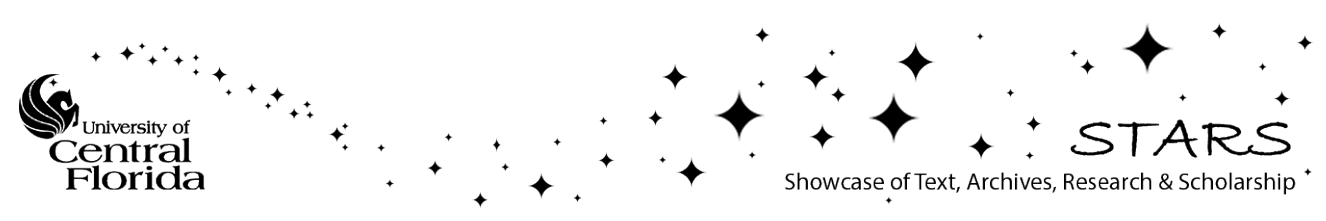




\section{Complex structure of carbon nanotubes and their implications for formation mechanism}

Cite as: Journal of Applied Physics 93, 9972 (2003); https://doi.org/10.1063/1.1573733

Submitted: 16 December 2002 . Accepted: 19 March 2003. Published Online: 30 May 2003

Dan Zhou, and Lee Chow

\section{ARTICLES YOU MAY BE INTERESTED IN}

Thermal physics in carbon nanotube growth kinetics

The Journal of Chemical Physics 121, 446 (2004); https://doi.org/10.1063/1.1755662

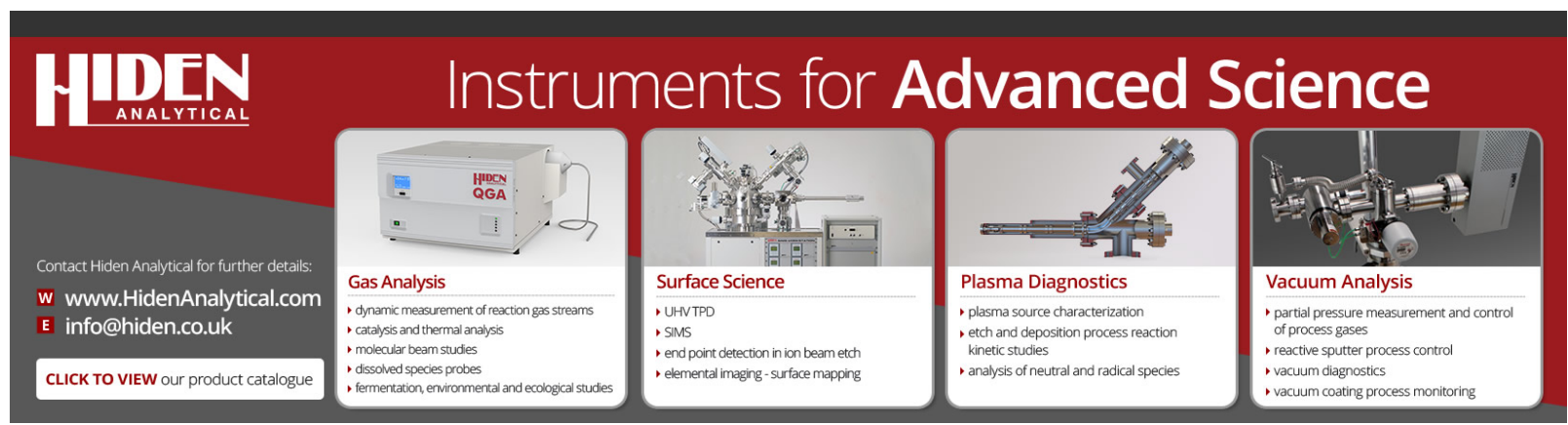




\title{
Complex structure of carbon nanotubes and their implications for formation mechanism
}

\author{
Dan Zhou \\ Department of Mechanical, Materials, and Aerospace Engineering, Advanced Materials Processing \\ and Analysis Center, University of Central Florida, Orlando, Florida 32816 \\ Lee Chow $^{\text {a) }}$ \\ Department of Physics, University of Central Florida, Orlando, Florida 32816
}

(Received 16 December 2002; accepted 19 March 2003)

\begin{abstract}
Complex structures of carbon nanotubes formed from a conventional carbon arc-discharge method have been revealed by high-resolution transmission electron microscopy (HRTEM). The branching and the droplet growth phenomena observed by HRTEM indicate that the formation and growth of carbon nanotubes from an arc-discharge method cannot be simply processed from one end to another. Based on the observation of the growth phenomena and the amorphous carbon residue inside the hollow core of carbon nanotubes, a two-step growth model of carbon nanotubes has been proposed. (c) 2003 American Institute of Physics. [DOI: 10.1063/1.1573733]
\end{abstract}

\section{INTRODUCTION}

The discovery and synthesis ${ }^{1,2}$ of carbon nanotubes have stimulated extensive studies for their potential application because of the unique mechanical and electronic properties of this class of materials. ${ }^{3-7}$ In order to realize their promising technological utilization, nanotubes with a desired morphology and microstructure must be prepared. This requires a control of the growth phenomena based on advanced knowledge of the nucleation and growth mechanisms of carbon nanotubes. This advanced knowledge is also critical to establishing the requirements for large-scale production. ${ }^{8,9}$ Therefore, a detailed study of the growth phenomena of carbon nanotubes will lead us to a better understanding of their formation and growth during a carbon arc-discharge evaporation.

The formation and growth mechanism of carbon nanotubes prepared by an arc discharge has been the subject of some controversy, particularly with regard to their microstructures. Based on high-resolution transmission electron microscopy (HRTEM) observations of open-ended nanotubes, Iijima et al. ${ }^{10}$ proposed an open-ended growth model in which carbon atoms and small clusters from the discharge plasma add on to the reactive dangling bonds at the edges of the open-ended nanotubes. This open-ended growth model suggests that during the growth process, the ends of nanotubes should be kept from closing by certain forces which are still unknown, and the walls of nanotubes are then concentrically thickened layer by layer from the inside to the outside along the same tubular axis. In contrast, Saito et al. ${ }^{11}$ proposed that during the discharge deposition, small carbon particles were first formed on the surface of the cathode, and these particles were elongated by an electrostatic force along the electric field just above the surface of the cathode to form the carbon tips. The tips collect carbon atoms from the plasma, and continually grow forward as long as carbon va-

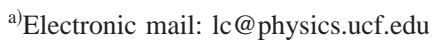

por and ions are supplied and the electric field is sustained. Meanwhile, the graphitization initiates from the bottom of the tips toward their end faces. Based on Saito et al.'s ${ }^{11}$ model, nanotubes in the cathodic deposit should be parallel and lined up along the direction of the electric field. Unfortunately, a rich variety of growth phenomena revealed by HRTEM characterizations indicate that the growth process of nanotubes from an arc discharge is complicated, and the current growth models cannot explain those growth behaviors. ${ }^{12,13}$

A better understanding of the formation and growth of carbon nanoclusters is imperative, if tailoring nanotubes into a desired structure for their advanced application is to be achieved. The basic elements of the growth dynamics should be known in order to systematically optimize the process parameters and control the nanotubes growth. Therefore, it is necessary to systematically investigate the growth phenomena and microstructure of nanotubes, and to expand our knowledge of their formation and growth. In this article, some of the microstructures and growth morphologies of carbon nanotubes from arc discharges have been reported based on an HRTEM analysis. According to the growth phenomena observed in this study, which show the sequential steps of the formation of carbon nanotubes, a two-step growth model that assumes graphitization of the final structures from an assembly of amorphous or disordered initial structures is proposed.

\section{EXPERIMENTAL PROCEDURES}

The samples used in this study were produced by a conventional arc-discharge method, which was operated at a current density of $220 \mathrm{~A} / \mathrm{cm}^{2}$ drawn by $27 \mathrm{~V}$ between the electrodes under a helium atmosphere of 500 Torr. The anode, being consumed during the discharge process, was a cylindrical graphite rod $0.64 \mathrm{~cm}$ in diameter and $30 \mathrm{~cm}$ in length. The cathode consisted of a graphite rod $0.95 \mathrm{~cm}$ in diameter and $3 \mathrm{~cm}$ in length. The gap between the two electrodes was maintained at approximately $1 \mathrm{~mm}$ during the operation. The 


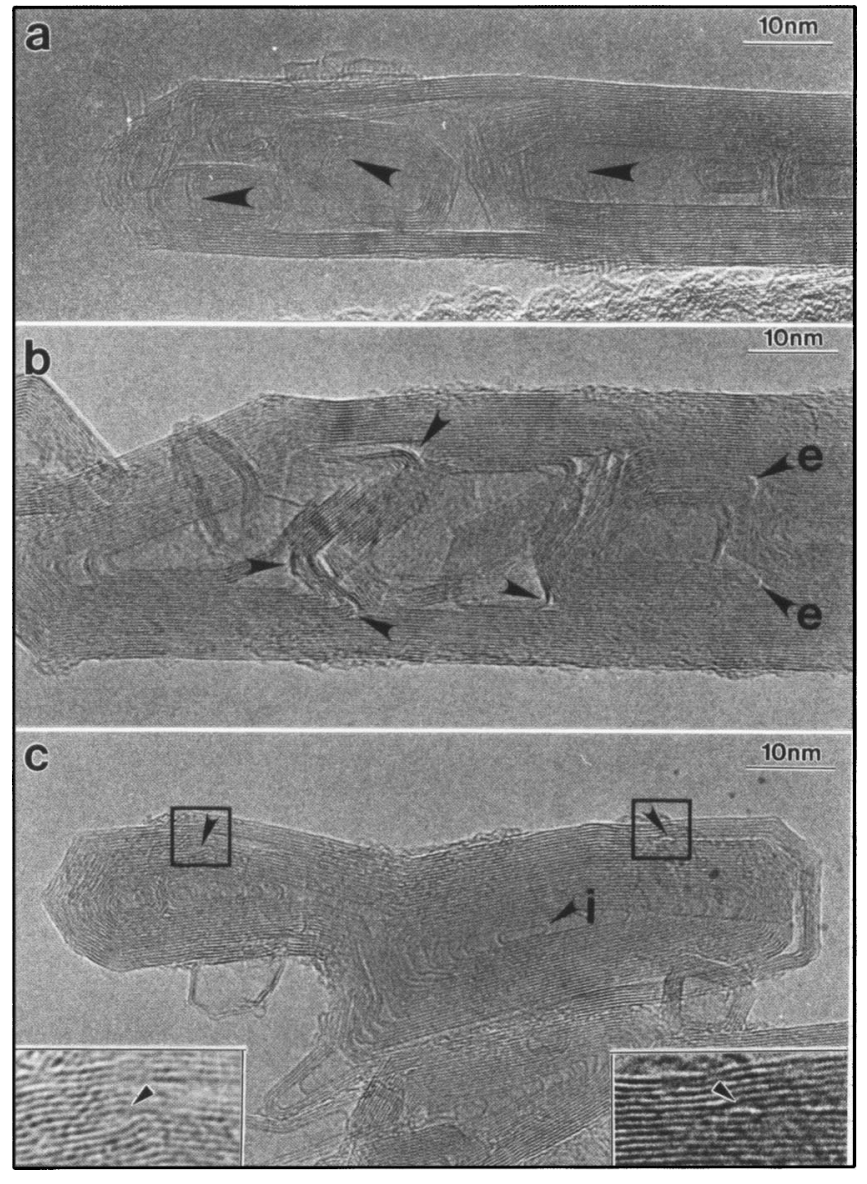

FIG. 1. (a) An HRTEM image shows that the nanotube contains multiple inner chambers (labeled by arrows) lined up by different axes. (b) A complex internal structure of the nanotube showing that the morphologies of the inner chambers are very flexible and depend on the confined space in which they are formed. (c) An HRTEM image indicates that the exterior walls of the nanocluster grow along different directions. The inset images show the edge dislocations in the graphitic walls.

carbonaceous deposit on the surface of the cathode was collected as a slag, and samples for a HRTEM analysis were prepared by grinding the initial slag into powder and dispersing the powder in ethanol or acetone. After sonicating the powder for approximately 5 min, the black suspension was dropped on a holey carbon thin film on a copper grid. The samples were then examined under a Hitachi H-8100 and a JEOL 2000 FX HRTEMS operated at $200 \mathrm{keV}$, respectively.

\section{RESULTS}

The carbonaceous materials (slag) deposited on the cathode of an arc discharge are composed of a mixture of different shapes of carbon nanotubes and nanoparticles besides the graphitic plates and amorphous carbon. Normally, carbon nanotubes consist of hollow carbon hexagonal networks concentrically arranged around one another, and terminated by end caps in which the surface meet under specific angles. Figure 1(a) shows a HRTEM image of the carbon nanotube containing multiple inner chambers, indicating that the cylindrical graphitic walls from different chambers are concentrically lined up along different axes (labeled by arrows). The existence of these chambers suggests that their nucleation

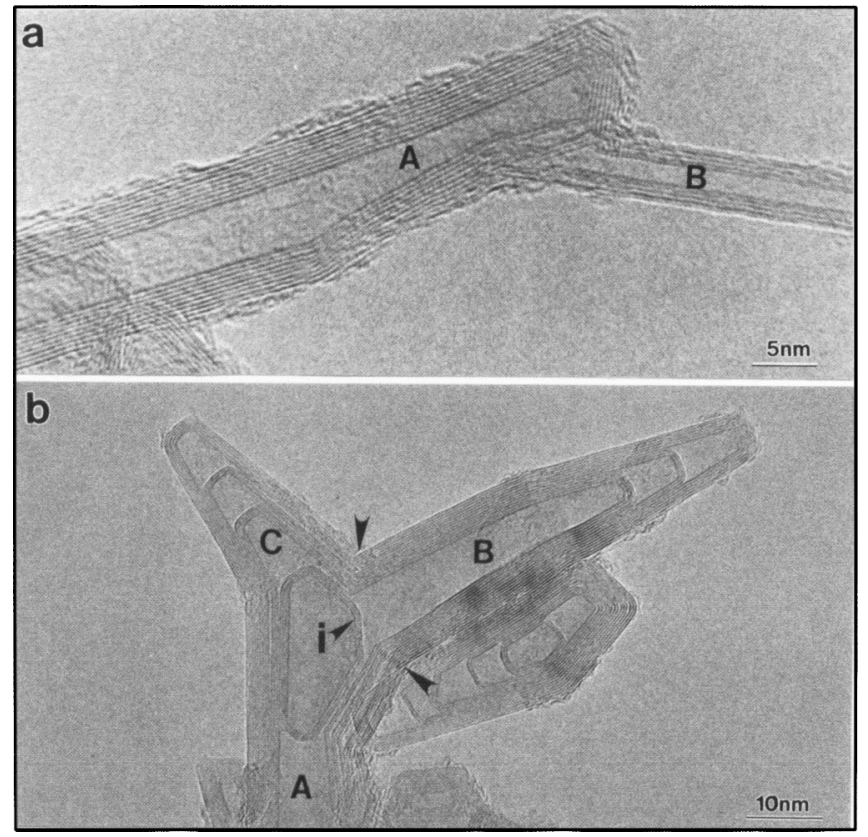

FIG. 2. (a) An HRTEM image shows that two nanotubes (A and B) are constructed together. (b) An HRTEM image demonstrates that three nanotubes $(\mathrm{A}, \mathrm{B}$, and $\mathrm{C})$ are joined under certain angles. The label $\mathrm{i}$ indicates the morphology of the innermost walls.

and formation processes are likely to occur from their outermost surface, which control the morphologies of the chambers, toward the inner in the confined space. A more complicated internal structure of a carbon nanotube, shown in Fig. $1(\mathrm{~b})$, demonstrates that the morphologies of the inner chambers are very flexible and depend on the confined space in which they are formed. This suggests that the graphitic walls are developed simultaneously through the entire carbon nanotube rather than initiated at one end and proceeded in one direction. The arrows in Fig. 1(b) show the flexible curvatures of the inner graphitic walls, and the label "e" indicate that the confined space results in linking two different graphitic walls together to avoid the dangling bonds rather than closing the wall by a cap. Moreover, Fig. 1(c) shows that the exterior walls of carbon nanocluster can also form along different directions, with the morphology of the nanocluster totally different from that of its innermost walls (labeled "i"). Again, this growth phenomenon suggests that the formation of the carbon nanocluster is likely to proceed from the surface toward the center, and that the morphology of the cluster is determined by the outer surface rather than by the innermost layers of the cluster. Notice that the layer structures of the carbon nanocluster are not perfect, and many different types of defects can be found during the growth process of the nanoclusters. Figure 1(c) also shows that the carbon nanocluster has two-dimensional defects indicated by arrows and their enlargement images are indicated as insets. These defects are similar to the projected image of an edge dislocation.

Figure 2(a) shows that a smaller nanotube (labeled "B") is grown out of the initial tubular base (labeled "A"), which indicates that the growth and formation of the tubular walls are not symmetrical along one tube axis. Furthermore, an- 


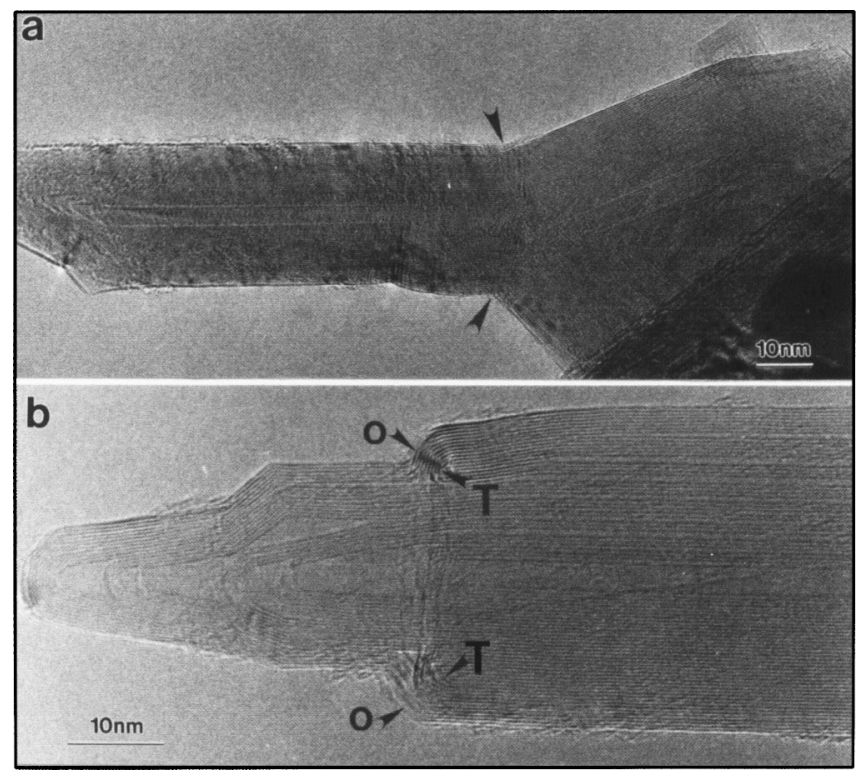

FIG. 3. (a) An HRTEM image shows that the carbon nanotubes and nanoparticle are connected with a droplet morphology. (b) An HRTEM image of carbon nanotube showing that the growth of the outermost walls (labeled "o") of the tube passes through the shoulder, but the inner walls are stopped there by joining two walls together.

other HRTEM image, shown in Fig. 2(b), demonstrates that three carbon nanotubes (labeled "A", "B", and "C") can be joined together to form a three-dimensional structure with a saddle surface, which has been predicted by theoretical calculations before. ${ }^{14-16}$ Notice that Fig. 2(b) clearly shows again that the morphology of the nanocluster does not follow that of the innermost walls (labeled "i") of the cluster. Furthermore, instead of the continual bending of the graphite layers, two sets of graphitic walls meet together under a certain angle [indicated by an arrow in Fig. 2(b)]. These growth phenomena indicate that an individual nanotube can grow simultaneously along different directions or axes, and the growth process of carbon nanotubes appears to proceed from the outer surface of nanoclusters.

In contrast to connecting the nanotubes together, an HRTEM image, shown in Fig. 3(a), illustrates a more complicated nanocluster structure, in which the nanocluster is constructed by both tubular and polyhedral structures with a droplet morphology. This droplet morphology indicates that the formation and growth of nanotubes and nanoparticles from a carbon arc discharge occur simultaneously. Notice that the arrows shown in Fig. 3(a) indicate the joint angle of the nanotube and nanoparticle components. Figure 3(b) shows an HRTEM image of carbon nanotube, demonstrating that the outer diameter of the nanotube is suddenly changed by stopping the further growth of several outer walls. The contrast of the lattice image (indicated by "T") suggests that the walls can be closed by linking two graphitic layers together through continual bending, in which one wall contributes a negative curvature and the other wall has a positive curvature. Notice that the growth of the outermost walls (labeled by "o") of the nanotube passes smoothly through the shoulder, but the inner walls are stopped by joining two walls

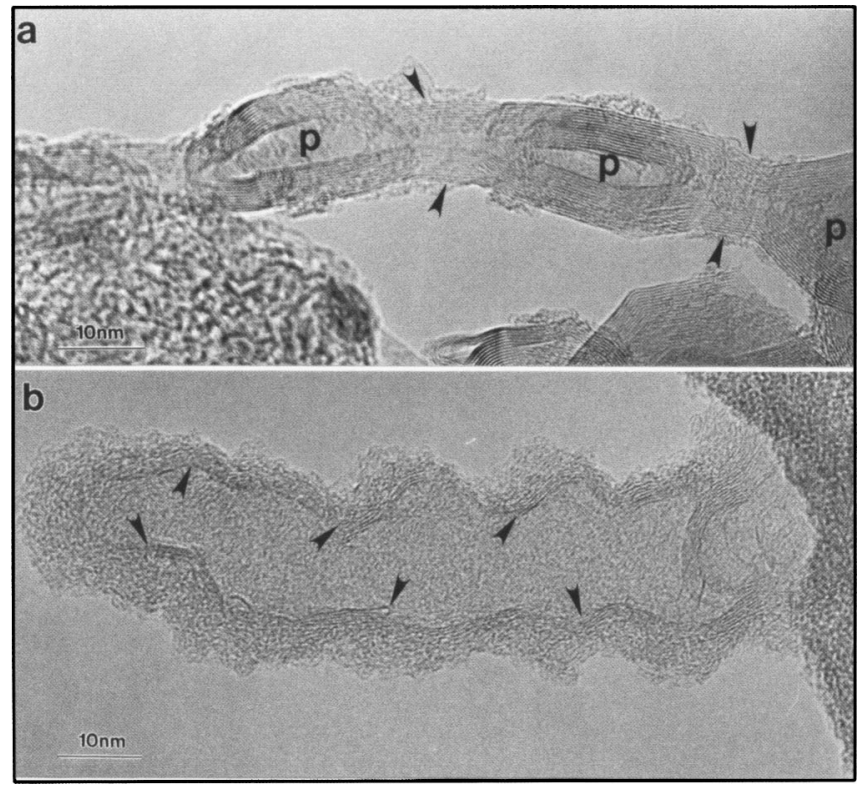

FIG. 4. (a) An HRTEM image showing that the elongated carbon nanoparticles (labeled "P") are aligned by continuous bent graphitic layers (labeled by arrows). (b) An HRTEM image showing an undeveloped graphitic nanocluster (or an assembly) carrying amorphous carbon. The arrows indicate the undeveloped graphitic layers in the assembly.

together, which also suggests that the outermost walls are formed first.

Furthermore, the elongated carbon nanoparticles (labeled by "P") can be constructed by continuously bent graphitic layers (indicated by arrows), shown in Fig. 4(a), illustrating that the graphitic walls should be initially developed on the surface of a carbon nanocluster rather than grown from an embryo. On the contrary, Fig. 4(b) shows an HRTEM image of an undeveloped graphitic nanocluster (or an assembly) which may be quenched as it is. In this nanocluster, the graphitic walls (indicated by arrows) are not well developed, and the cluster consists mostly of disordered graphite and amorphous carbon. The observation of such a disordered carbon assembly supports the speculation that graphitic nanoclusters are developed through the graphitization process from the amorphous carbon assemblies produced by carbon discharge plasma. The morphologies of the nanotubes are therefore predetermined by the shapes of the amorphous carbon assemblies.

More evidence to support this speculation is provided in Fig. 5. An HRTEM image of a nanotube [see Fig. 5(a)] demonstrates that amorphous carbon residue is left either inside the hollow core (indicated by arrows) or on the outer surface (labeled "S") of the nanotube. Further details are shown in Fig. 5(b). The contrast of Fig. 5(b) indicates that the hollow core of the tube is occupied by amorphous carbon, and that the discontinued inner walls (shown by arrows) have a fairly lower degree of graphitic order than that in the peripheral region. Notice that the label "d" clearly shows a projected image of edge dislocations in the layer structure of carbon nanotubes.

Figure 6(a) shows that a cylindrical amorphous assembly is just partially graphitized. According to this HRTEM image, in Fig. 6(a), the assembly has well-developed graphitic 


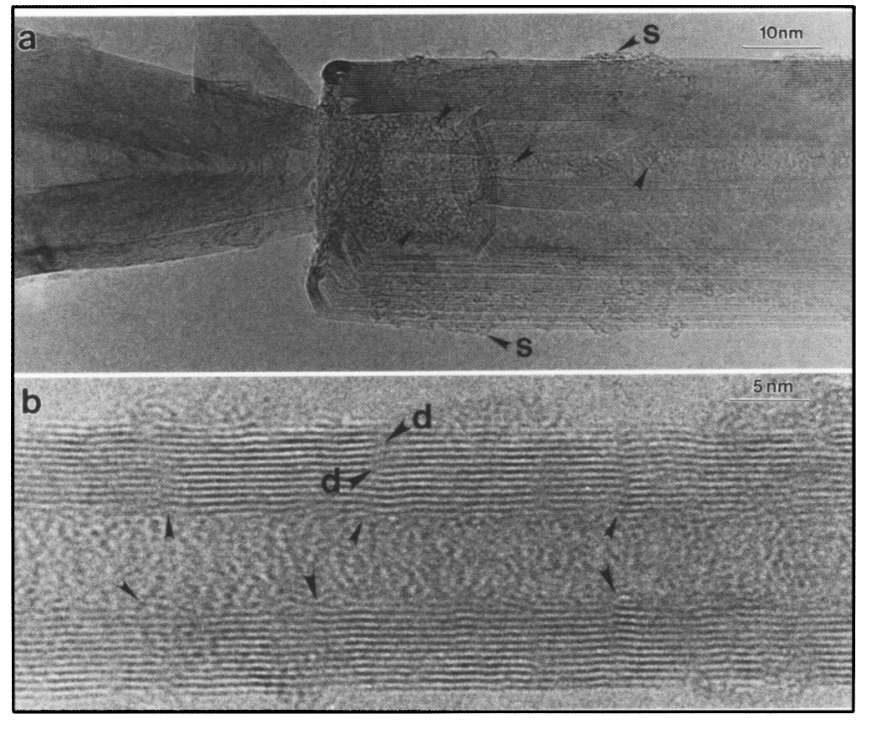

FIG. 5. (a) An HRTEM image of a quenched nanotube showing that the disordered carbon residue is left either inside the hollow core (indicated by arrows) or on the outer surface (labeled by "S"). (b) An HRTEM image indicates that the hollow core of the tube is occupied by disordered carbon, and that the inner walls (shown by arrows) have a fairly lower degree of graphitic order than that in the peripheral region. The label "d" illustrates a pair of edge dislocations on one side of the graphitic walls of the nanotube.

walls (labeled " $T$ ") at the outer surface, but amorphous carbon (labeled by "A") and graphitic layers (labeled by "G") are encaged inside the nanotube. Note that the graphitic layers inside the tube have not been aligned along the tube axis yet, and the graphitization process has been interrupted for some reasons. Figure 6(b) shows an HRTEM image of a joint of tow nanotube components (labeled by " $T$ "). The image in Fig. 6(b) demonstrates that following the morphology of the joint, several graphitic layers (labeled by "G") have been developed on the outermost surface, and then the graphitization process has been stopped since there is amorphous carbon (labeled by "A") residing in between the nanotubes and

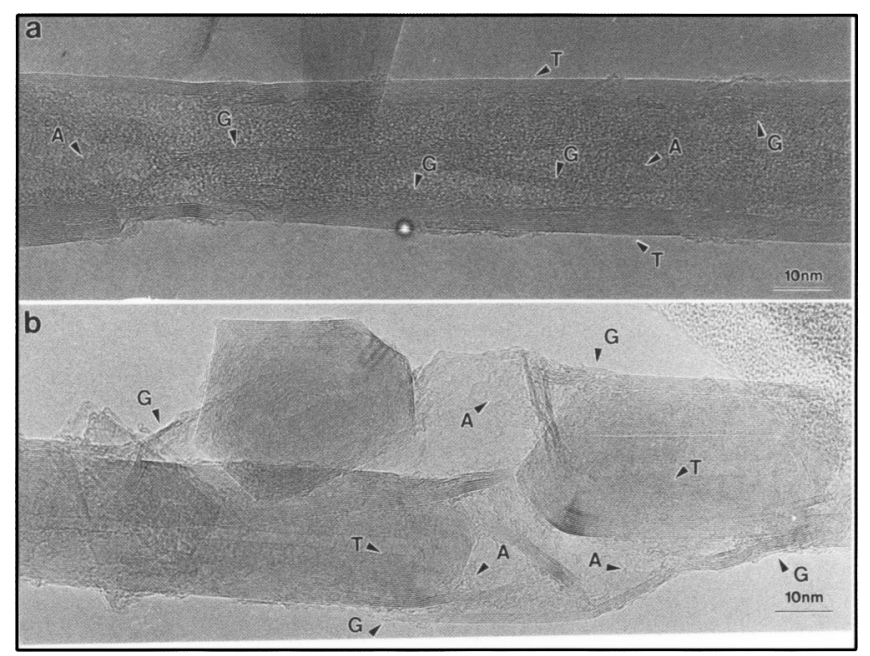

FIG. 6. HRTEM images shows (a) a cylindrical amorphous assembly, which is just partially graphitized and (b) a joint of two nanotube components. The outer graphitic walls are labeled by " $\mathrm{T}$ ", and amorphous carbon and graphitic layers inside the tube are indicated by "A" and "G". graphitic layers. These results simply indicate that the graphitic tubes were initiated from the amorphous carbon assemblies. The graphitization of these amorphous carbon assemblies toward the center has been interrupted for some reasons, such as quenching or fluctuation of the discharge plasma.

\section{DISCUSSION}

The rich variety of carbon nanotube structures as observed here by the HRTEM analysis suggests that the formation of carbon nanotubes is a complex process. The difficulties of interpreting the inner and outer structure of nanotubes can be reduced by assuming that the formation of carbon nanotubes proceeds in two steps. First, amorphous carbon assemblies are formed on the surface of the cathode (hightemperature region in excess of $3500{ }^{\circ} \mathrm{C}$ ) during the arcdischarge evaporation. These assemblies may have different shapes, such as plate, sphere, polyhedron, droplet, and cylinder, with random orientation, which depend on their surface energy and the local discharge conditions. Note that under certain arc-discharge conditions, the cylindrical amorphous assemblies may be preferred. Second, during the cooling process, the graphitization consisting of joining small layer plane segments together to form larger plane areas and then rotating and translating these near-perfect layers into proper stacking, occurs simultaneously on the assemblies from the surface toward the interior region. The growth of the concentric graphite walls with cooling first occurs near the surface. This is then followed by further alignment and joining of the internal crystallites to the outer shell. Sometime the misjoining of the graphitic walls causes the edge dislocations. Since the density of amorphous carbon assemblies is varied and smaller than that of graphite, in most of the cases, the empty cores are left inside the graphitic nanoclusters when the graphitization starts on the surface of the assemblies. The outer diameter of the tubes is determined by the diameters of the assemblies, and the inner diameter of the tubes or the size of the hollow cores depends on the difference of the densities between the amorphous carbon assemblies and graphite networks. Notice that the growth tends to be confined within the boundaries of the primary carbon nanoclusters aggregates, with most layer planes aligning parallel to the surface of the clusters. Those layer planes, which cannot be aligned parallel to the surface of the clusters, will cap the walls to form the inner chambers.

It is important to note that since the formation of the graphitic walls may result from the graphitization of the assemblies, the introduction and distribution of the pentagons and heptagons in the hexagonal network will certainly depend on the morphologies of the assemblies, which are controlled by their surface energy and the local discharge conditions. According to the growth model of carbon nanotubes proposed here, it is not necessary to speculate a force which keeps the ends of nanotubes open for forward growth because carbon nanotubes are developed from the cylindrical amorphous carbon assemblies through the graphitization process. Furthermore, the fluctuation of the carbon arc discharge may be avoided if one can produce the needlelike carbon 
assemblies in the nanometer scale at a high temperature by alternate methods, such as vapor condensation of carbon, ${ }^{17}$ plasma decomposition of hydrocarbon, ${ }^{18}$ and flames of acetylene and benzene (or ethylene) premixed with oxygen, ${ }^{19}$ and then carbon nanotubes can be formed through a cooling process.

\section{CONCLUSION}

Complex structures of carbon nanotubes produced by the conventional arc-discharge method have been characterized by HRTEM. In these structures, the growth of graphitic walls of carbon nanotubes are not always concentrically aligned. The morphologies of the inner chambers of nanotubes are very flexible and dependent upon the confined space in which they are formed. The branching and the droplet growth phenomena have also been observed, indicating that the formation and growth of carbon nanotubes from an arcdischarge method cannot be simply processed from one end to another. Furthermore, amorphous carbon has been frequently observed in the hollow cores of the nanotubes. Based on these growth phenomena and the amorphous carbon residue inside the hollow core of some nanotubes, a two-step growth mechanism of carbon nanotubes has been proposed. First, the amorphous carbon assemblies with any kind of morphology, such as plate, sphere, polyhedron, droplet, and cylinder with random orientation, which depends on their surface energy and the local discharge conditions, are formed on the cathode surface (high-temperature region). Second, during the cooling process, the graphitization occurs from the surface toward the interior of the assemblies. The morphologies of carbon nanoclusters appear to be solely determined by the shapes of the amorphous assemblies and that the needlelike assemblies, which result in the tubular structures after the graphitization, are preferred under some discharge conditions.

\section{ACKNOWLEDGMENTS}

One of the authors (D. Z.) acknowledges S. Seraphin and B. Seraphin of the University of Arizona for valuable discussions and support, and J. C. Withers of Materials Electrochemical Corp. Tucson, Arizona, for providing the samples for this study. He also acknowledges support from the Advanced Materials Processing and Analysis Center and the Material Characterization Facility at the University of Central Florida.

${ }^{1}$ S. Iijima, Nature (London) 354, 56 (1991).

${ }^{2}$ T. W. Ebbesen and P. M. Ajayan, Nature (London) 358, 220 (1992).

${ }^{3}$ M. S. Dresselhaus, Nature (London) 358, 195 (1992).

${ }^{4}$ R. E. Smalley, Mater. Sci. Eng., B 19, 1 (1993).

${ }^{5}$ H. Dai, J. H. Hafner, A. G. Rinzler, D. T. Colbert, and R. E. Smalley, Nature (London) 384, 147 (1996).

${ }^{6}$ P. G. Collins, A. Zettl, H. Bando, A. Thess, and R. E. Smalley, Science (Washington, DC, U.S.) 278, 100 (1997).

${ }^{7}$ M. M. Treacy, T. W. Ebbesen, and J. M. Gibson, Nature (London) 381, 678 (1996).

${ }^{8}$ V. P. Dravid, X. Lin, Y. Wang, X. K. Wang, A. Yee, J. B. Ketterson, and R. P. H. Chang, Science (Washington, DC, U.S.) 259, 1601 (1993).

${ }^{9}$ S. Seraphin, D. Zhou, and J. Jiao, Carbon 31, 1212 (1993).

${ }^{10}$ S. Iijima, P. M. Ajayan, and T. Ichihashi, Phys. Rev. Lett. 69, 3100 (1992).

${ }^{11}$ Y. Saito, T. Yoshikawa, M. Inagaki, M. Tomita, and T. Hayashi, Chem. Phys. Lett. 204, 277 (1993)

${ }^{12}$ S. Seraphin, D. Zhou, and J. Jiao, Acta Microscopia 3, 45 (1994).

${ }^{13}$ D. Zhou and S. Seraphin, Chem. Phys. Lett. 238, 286 (1995).

${ }^{14}$ A. L. Mackay and H. Terrones, Nature (London) 352, 762 (1991).

${ }^{15}$ T. Lenosky, X. Gonez, M. Teter, and V. Elser, Nature (London) 355, 333 (1992).

${ }^{16}$ L. A. Chernozatonskii, Phys. Lett. A 172, 173 (1992).

${ }^{17}$ M. Ge and K. Sattler, Science (Washington, DC, U.S.) 260, 515 (1993).

${ }^{18}$ N. Hatta and K. Murata, Chem. Phys. Lett. 217, 398 (1994).

${ }^{19}$ J. B. Howard, K. das Chowdhury, and J. B. van de Sande, Nature (London) 370, 603 (1994). 